\title{
Lightpath Establishment Assisted by Offline QoT Estimation in Transparent Optical Networks
}

\author{
Nicola Sambo, Yvan Pointurier, Filippo Cugini, Luca Valcarenghi, Piero Castoldi, and Ioannis Tomkos
}

\begin{abstract}
We propose lightpath establishment techniques for transparent optical networks, valid for both centralized path computation (i.e., performed by a path computation element-PCE) and distributed path computation (i.e., performed by each ingress node). The proposed techniques rely on correlating the end-to-end quality of transmissions (QoT) (e.g., optical-to-signal-noise ratio-OSNR) of lightpaths already set up to evaluate the QoT of lightpaths to be established. The correlation is leveraged by two end-to-end estimation frameworks called "network kriging" and "norm $\ell_{2}$ minimization." Each framework is used in turn to estimate end-to-end parameters that describe the QoT for a lightpath to be established, based on measured parameters from other lightpaths that were previously established or probed. Simulations are carried out for both PCE and distributed networking scenarios on a sample optical transparent network. The proposed lightpath establishment techniques reduce the probability that a setup attempt is unsuccessful due to unacceptable QoT and reduce the number of setup attempts to successfully establish lightpaths. Simulation results are also provided to show that our techniques permit a fast convergence of QoT information at PCE or ingress nodes in the context of dynamic networks.
\end{abstract}

Index Terms-QoT; Transparent optical networks; PCE; GMPLS; Network tomography.

\section{INTRODUCTION}

D ynamic transparent optical networks, where lightpaths are provisioned and established on demand, are widely seen as the key enabling technology for the core network of the future [1]. Indeed, in such networks, the removal of optical/electrical/optical (OEO) conversion can decrease both capital expenditures (CAPEX) and energy consumption at the same time [2], while the increased available bandwidth and flexibility can decrease operational expenditures (OPEX).

Manuscript received March 2, 2010; revised August 19, 2010; accepted August 25, 2010; published October 20, 2010 (Doc. ID 124942).

N. Sambo (e-mail: nicola.sambo@sssup.it), L. Valcarenghi, and P. Castoldi are with Scuola Superiore Sant'Anna, Via G. Moruzzi, 1, 56124 Pisa, Italy.

At the time this work was performed, Y. Pointurier was with Athens Information Technology, 19.5 klm, Markopoulo Avenue, GR - 19002, Peania Attikis, Athens, Greece.

F. Cugini is with CNIT, Via G. Moruzzi, 1, 56124 Pisa, Italy.

I. Tomkos is with Athens Information Technology, $19.5 \mathrm{klm}$, Markopoulo Avenue, GR - 19002, Peania Attikis, Athens, Greece.

Digital Object Identifier 10.1364/JOCN.2.000928
A drawback of transparency is the increase of physical propagation distances over which physical layer impairments accumulate, potentially resulting in unacceptable lightpath quality of transmission (QoT) [3], typically measured in terms of the bit error rate (BER). In transparent optical networks controlled by generalized multiprotocol label switching (GMPLS), path computation may be either distributed, i.e., performed at each ingress node, or centralized, e.g., performed by a path computation element (PCE) [4]. A PCE is an entity capable of computing paths requested from path computation clients (PCCs) based on a network graph, applying, if needed, computational constraints, for instance, on bandwidth and QoT.

Since QoT must be satisfied along a path, several techniques have been proposed to evaluate it. QoT can be evaluated through estimation or measurements (monitoring). Analytical models can estimate QoT, before a lightpath is established, based on the prior knowledge of network physical parameters (evaluated at network installation time or through live monitoring) [3,5-10]. In the case of QoT estimation, mechanisms are required to provide either the centralized PCE or the ingress nodes with updated QoT information. Extensions to the PCE architecture [11] or to the GMPLS protocol suite (e.g., to the routing protocol [12] or the signaling protocol [13]) have been proposed to encompass QoT information. In the case of centralized PCE architecture, thanks to the centralized nature of the path computation, effective traffic engineering (TE) solutions are potentially achievable. However, additional mechanisms to provide the PCE with QoT information are required, e.g., dedicated communication between monitors and the PCE, as discussed in [11]. In the case of GMPLS routing protocol extensions, QoT parameters are flooded within the entire transparent optical network through the OSPF-TE protocol. On the one hand, this enables each source (i.e., ingress) node to estimate lightpath QoT along any possible route toward each destination. On the other hand, this may affect control plane scalability and convergence performance. In the case of GMPLS signaling protocol extensions, QoT information is included within RSVP-TE signaling messages. Thus, each node becomes aware of only the QoT information associated with lightpaths for which the node is either a transit or an end point. On the one hand, with respect to routing protocol extensions, this improves control plane performance scalability. On the other hand, signaling protocol extensions may prevent lightpath QoT estimation before lightpath setup due to lack of QoT information at a source 
node. In this case, a lightpath setup attempt is required for retrieving lightpath QoT information and performing QoT estimation. If the estimated QoT is unacceptable, the lightpath setup is blocked and another attempt might take place, delaying the lightpath setup. The Internet Engineering Task Force (IETF) is more inclined to encompass QoT information within the PCE architecture or the GMPLS signaling protocol and not within the routing protocol for scalability reasons [14]. For this motivation, in this paper we concentrate on both the PCE-based and the signaling-based solutions.

When the QoT is evaluated through measurements, monitoring techniques can be used during the lightpath establishment to assess more directly whether the signal's QoT is acceptable [15-17]. Measurements on probe traffic aim at removing any inaccuracy stemming from analytical models. For this reason, probing is also considered in the paper. In particular in [16], a lightpath establishment scheme is assisted by monitoring and probing, in which probe traffic is sent and the BER is measured just before lightpath activation (i.e., before client data transmission). However, with probing, the lightpath establishment could be delayed. Indeed, if the measured QoT is unacceptable, the lightpath establishment is blocked and another attempt might take place, increasing the setup delay and wasting the resources reserved before probing. Therefore, lightpath establishment techniques are required that guarantee the QoT with limited knowledge of QoT information and reduce the number of establishment attempts.

In this paper, lightpath establishment techniques exploiting both QoT estimation and monitoring are proposed for distributed and centralized (i.e., PCE-based) path computations. Monitoring information is exploited by QoT estimation, which allows lightpath establishment to speed up by avoiding establishment attempts along paths that are infeasible in terms of QoT. Hence, we propose to infer or estimate QoT parameters for lightpaths, given the knowledge of monitoring data previously collected for other lightpaths. Here, we propose to apply two estimation techniques, called "network kriging" [18] and "norm $\ell_{2}$ minimization" (i.e., $\ell_{2}$-min) [19], typically used in "network tomography" [20], in the context of dynamic transparent optical networks. The two estimation frameworks leverage the correlation between QoT parameters related to lightpaths with common links. The PCE, in the PCE-based scenario, or the ingress node, in the distributed scenario, compute the paths to route the lightpath that has been requested. The PCE (or the ingress node) exploits available past probing data to estimate a new lightpath's QoT through network kriging or $\ell_{2}$-min. If the estimated QoT is acceptable, probing is performed to verify the lightpath QoT; otherwise, another path is computed. We show through simulations that the establishment techniques assisted by network kriging and $\ell_{2}$-min decrease the probability that a lightpath establishment attempt is unsuccessful and thus reduce the number of successive attempts to successfully establish lightpaths in both network scenarios. We also assess with simulations that our techniques allow a fast convergence of estimated QoT parameters at each ingress (or PCE) node, an important property in the context of dynamic network scenarios where physical parameters may change with time and where management algorithms must adapt and react to changing conditions.

\section{ESTIMATION FRAMEWORKS}

In this section, we present the two estimation frameworks that we utilize to evaluate lightpaths' QoT based on the monitoring data collected during previous lightpath establishments. The two techniques are, namely, "network kriging" (further denoted by NK) [18], and a technique based on "norm $\ell_{2}$ minimization" (further denoted by $\ell_{2}$-min) [19]. Both techniques rely on the correlation between end-to-end parameters that is induced by a network topology: the QoT parameters of lightpaths sharing a large number of links are highly correlated, while there is no correlation between the QoT of lightpaths that do not share any link.

In this study we consider four end-to-end parameters, which describe QoT, accounting for the following transmission impairments: amplified spontaneous emission (ASE), polarization mode dispersion (PMD), chromatic dispersion (CD), and self-phase modulation (SPM). Note that other effects (such as cross-phase modulation-XPM, four-wave mixing-FWM) are considered with fixed worst-case margins (i.e., all channels lit) as is typically done by operators in current networks. Fixed worst-case margins are computed in the worst-case scenario during network installation considering the set of possible routes and all channels lit. Thus, fixed worst-case margins are applied during lightpath provisioning independently on the lightpath route. If a lightpath has acceptable QoT in the worst-case scenario, its QoT will be certainly acceptable also when new lightpaths are established. In this paper, a single bit-rate network (e.g., $10 \mathrm{Gbit} / \mathrm{s}$ ) is considered, however, in the case of XPM in multi-bit-rate networks, worst-case margins could be too pessimistic so that considering a guard band among interfering lightpaths can be a better solution than assuming worst-case margins [21].

The estimation frameworks assume the existence of a linear relationship between the link-level parameters and the end-to-end parameters to be estimated. In the considered optical networking context, linearity holds for each of the four aforementioned physical impairments; specifically,

- OSNR degradations are caused by the accumulation of the ASE noise; the inverse of OSNR is linear, i.e., by denoting with $r_{i}$ the OSNR parameter of a lightpath using only the link $i$, the OSNR $R_{\ell}$ of a lightpath $\ell$ consisting of two consecutive links $i$ and $j$ is such that $1 / R_{\ell}=1 / r_{i}+1 / r_{j}$

- denoting by $p_{i}$ the average PMD of link $i$ (in ps), the PMD $P_{\ell}$ accumulated along a lightpath $\ell$ is such that $P_{\ell}^{2}=p_{i}^{2}+p_{j}^{2}$. Hence, the square of the average PMD accumulates linearly;

- CD accumulates linearly; denoting by $d_{i}$ the dispersion parameter of link $i$ (in ps/nm), $D_{\ell}=d_{i}+d_{j}$;

- SPM can be quantified through the nonlinear phase of the signal $\varphi$, which accumulates linearly [22]: $\Phi_{\ell}=\varphi_{i}$ $+\varphi_{j}$.

Note that each of the four end-to-end parameters $R, P, D$, and $\Phi$ can be monitored by using the appropriate hardware: an OSNR monitor [15,23,24] for $R$; PMD [23,25-27] and CD [28-30] monitors for $P$ and $D$, respectively; and a power 
monitor for $\Phi[22]$, making the QoT estimation via the estimation of OSNR, PMD, CD, and nonlinear phase practical. Regarding nonlinear phase, $\Phi$ is computed by considering that fiber nonlinear parameters (e.g., effective area- $A_{\text {eff }}$ ) are roughly constant and known for fibers from a given type [31,32], and an overestimate value can be used for each kind of fiber in the network. Then, the model in [13] is utilized to derive the lightpath QoT from $R, P, D$, and $\Phi . D$ and $\Phi$ parameters are computed as a penalty to the OSNR. Then, penalty and worst-case margins (to account for nonmodeled effects as XPM) are subtracted from $R$ to compute the final $R_{f}$. Finally, the lightpath QoT is estimated to be acceptable if i) $R_{f}$ is higher than a threshold that guarantees a maximum BER (e.g., $10^{-9}$ after forward error correction), and ii) the $P$ parameter is below a threshold (e.g., $10 \%$ of the bit duration).

\section{A. Notation}

Consider a network with $E$ (unidirectional) links and where $L$ lightpaths are established. The network is abstracted through the routing matrix $G \in\{0,1\}^{L \times E}$, where $G_{\ell, j}=1$ when lightpath $\ell$ uses link $j$. Consider the end-to-end parameter values $\mathbf{y} \in \mathbb{R}^{L}$, where $y_{\ell}$ is a value for lightpath $\ell$. We assume in addition that the end-to-end parameters $\mathbf{y}$ can be written as linear combinations of link-level parameters $\mathbf{x} \in \mathbb{R}^{E}$. In particular, we assume that parameter $y_{\ell}$ is equal to the sum of the parameters $x$ over all links used by lightpath $\ell$, that is, $\mathbf{y}=G \mathbf{x}$. The end-to-end QoT parameters y that we consider correspond to either $1 / R, P^{2}, D$, or $\Phi$, while the link-level QoT parameters $x$ correspond to either $1 / r, p^{2}, d$, or $\varphi$.

We now assume that some of the end-to-end values in $\mathbf{y}$ are already known through measurements, while others are not. We reorder the values within $\mathbf{y}$ and the rows of $G$ as follows. Denote with $\mathbf{y}_{m}$ the parameters of the lightpaths for which monitoring data are available and with $\mathbf{y}_{n}$ the parameters for the lightpaths for which monitoring data is not available and that should be estimated: $\mathbf{y}=\left[\mathbf{y}_{m}^{T}, \mathbf{y}_{n}^{T}\right]^{T}$. Similarly, denote with $G_{m}$ the matrix formed by the rows of $G$ that correspond to lightpaths for which monitoring information is available and $G_{n}$ the rows of $G$ corresponding to lightpaths for which no monitoring information is available, such that $G=\left[G_{m}^{T}, G_{n}^{T}\right]^{T}$. Then

$$
\mathbf{y} \triangleq\left[\begin{array}{l}
\mathbf{y}_{m} \\
\mathbf{y}_{n}
\end{array}\right]=\left[\begin{array}{l}
G_{m} \\
G_{n}
\end{array}\right] \mathbf{x} .
$$

Consider for instance the network depicted in Fig. 1, where end-to-end parameters $\mathbf{y}_{m}=\left[y_{1}, y_{2}, y_{3}\right]^{T}$ for lightpaths LP1, LP2, and LP3 are known (lightpaths may still be present in the network, or they may have already been torn down). If the demand for a fourth lightpath LP4 arrives, restricting our view of the network to the three links L1, L2, and L3 with respective link-level parameters $\mathbf{x}=\left[x_{1}, x_{2}, x_{3}\right]^{T}$, we have

$$
\left[\begin{array}{l}
y_{1} \\
y_{2} \\
y_{3} \\
y_{4}
\end{array}\right]=\left[\begin{array}{llll}
1 & 0 & 0 & 1 \\
0 & 0 & 1 & 0 \\
0 & 1 & 1 & 0 \\
1 & 1 & 0 & 0
\end{array}\right]\left[\begin{array}{l}
x_{1} \\
x_{2} \\
x_{3} \\
x_{4}
\end{array}\right] .
$$

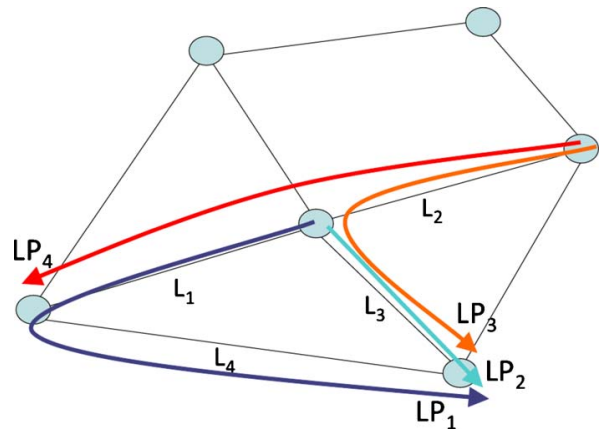

Fig. 1. (Color online) Illustrative example: estimation of the endto-end properties of lightpath LP4 given the knowledge of the endto-end properties of lightpaths LP1, LP2, and LP3 obtained through monitoring.

Because all four lightpaths share the same set of links, it is possible to estimate $\mathbf{y}_{n}=y_{4}$ given $y_{1}, y_{2}$, and $y_{3}$ and the routing matrix $G$, using network kriging or $\ell_{2}$-min. We show how this is done in the following subsection.

\section{B. Network Kriging and Norm $\ell_{2}$ Minimization}

Consider the following general estimation problem: given a routing matrix $G=\left[G_{m}^{T}, G_{n}^{T}\right]^{T}$, the end-to-end observations $\mathbf{y}_{m}=G_{m} \mathbf{x}$, where the link-level parameters $\mathbf{x}$ are unknown, determine all end-to-end parameters $\mathbf{y}_{n}$, where $\mathbf{y}_{n}=G_{n} \mathbf{x}$. Network kriging and norm $\ell_{2}$ minimization approximate $\mathbf{y}_{n}$ as follows.

\section{- Network kriging (NK):}

Using the notations above, the best (in terms of mean-square error) linear estimate $\hat{\mathbf{y}}_{n}$ for the parameters corresponding to nonobserved lightpaths can be shown, as in [18], to be

$$
\hat{\mathbf{y}}_{n}=G_{n} G_{m}\left(G_{n} G_{m}^{T}\right)^{+} \mathbf{y}_{m},
$$

where $(\cdot)^{+}$denotes a pseudoinverse such as the MoorePenrose inverse.

This estimator is presented in [18], together with an assessment of the scalability performance of the method: with NK, the computation time is dominated by matrix inversion operations and the complexity is $O\left(M^{3}\right)$, where $M$ is the number of observed lightpaths. Thus, NK is particularly suitable in the case of a limited amount of observations, while it may suffer from scalability performance in the case of a large amount of monitoring information.

- Norm $\ell_{2}$ minimization $\left(\ell_{2}\right.$-min):

It is also possible to seek nonlinear estimates of $\mathbf{y}_{n}$ although they can be more computationally intensive than linear estimates. In particular, one technique to estimate $\mathbf{y}_{n}$ consists of first finding an estimate of $\mathbf{x}$ verifying some property (such as $\mathbf{x}$ having a minimum for some norm). Several norms can be a candidate for such minimization, but a particularly popular choice is the minimization of the $\ell_{2}$ norm. In this case the aforementioned $\mathbf{y}_{n}$ estimation problem is equivalent to the problem of finding the solution $\mathbf{x}$ to the following minimization problem [19]:

$$
\underset{\mathbf{x}, r}{\min }\|\mathbf{x}\|_{2}^{2}+\|r\|_{2}^{2}
$$




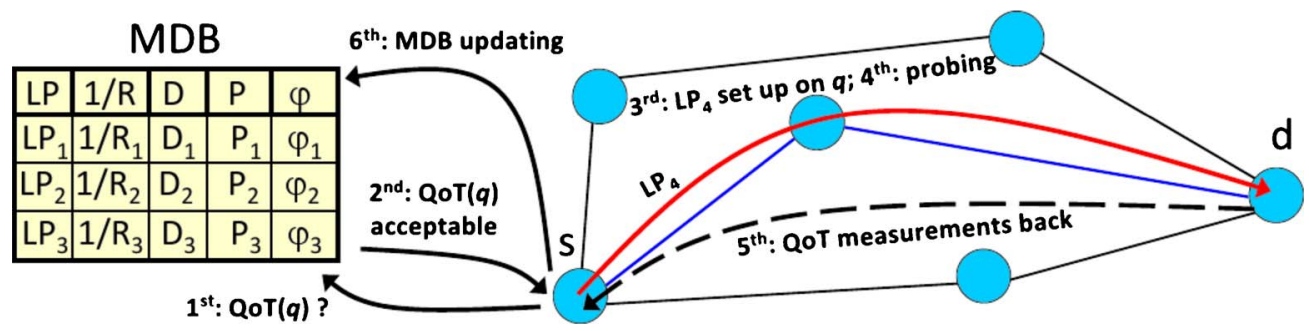

Fig. 2. (Color online) Lightpath establishment operations in a distributed scenario with D-NKS and D-LMS.

subject to $G_{m} \mathbf{x}+D_{2} r=\mathbf{y}_{m}, \quad 0 \leqslant \mathbf{x} \leqslant \max \mathbf{y}_{n}$.

In Eq. (4), $r$ is a regularization parameter and $D_{2}$ is a column vector indicating the relative accuracy ${ }^{1}$ required for satisfying each row of $G_{m} \mathbf{x}+D_{2} r=\mathbf{y}_{m}$. The problem solution returns an estimate $\hat{\mathbf{x}}$ of the link-level parameters $\mathbf{x}$; in this work, we obtain the estimates of $\mathbf{y}_{n}$ simply by using the relation $\hat{\mathbf{y}}_{n}=G_{n} \hat{\mathbf{x}}$. The constraint $0 \leqslant \mathbf{x} \leqslant \max \mathbf{y}_{n}$ ensures that the estimated link-level parameters $\mathbf{x}$ are nonnegative ${ }^{2}$ and that no link-level parameter can be greater than the largest end-to-end parameter (since end-to-end parameters are sums of link-level parameters). Problem (4) is thus a nonnegative linear least-squares problem, which can be solved by using software packages such as PDCO [33]. In terms of scalability, the $\ell_{2}$-min complexity is $O\left(a E^{3}\right)$, where $a$ depends on the required accuracy on the estimates of $\mathbf{x}$ in Eq. (4) [34]. Thus, with $\ell_{2}$-min, the path computation time is dominated by the number of links in the network.

In the estimation procedure, we estimate the QoT for unobserved lightpaths given the QoT of the observed lightpaths and information about the (spatial) correlation structure between the QoT parameters for different lightpaths. The existence of the linear relationships $\mathbf{y}_{m}=G_{m} \mathbf{x}$ and $\mathbf{y}_{n}$ $=G_{n} \mathbf{x}$ ensures that we can use the NK and $\ell_{2}$-min procedures to estimate $\mathbf{y}_{n}$ given $G_{n}, G_{m}$, and $\mathbf{y}_{m}$. This estimation procedure is run in turn for $\mathbf{R}, \mathbf{P}, \mathbf{D}$, and $\Phi$. QoT is then computed from the estimated parameters as described in Section II.

\section{LIGHTPATH ESTABLISHMENT EXPLOITING ESTIMATION FRAMEWORKS}

Four lightpath establishment techniques are proposed:

- The lightpath establishment with distributed network kriging framework scheme (D-NKS) is proposed for a distributed path computation scenario, and it exploits the NK framework (see Subsection III.A).

- The lightpath establishment with distributed $\ell_{2}$-min framework scheme (D-LMS) is proposed for a distributed path computation scenario, and it exploits the $\ell_{2}$-min framework (see Subsection III.A).

- The lightpath establishment with centralized network kriging framework scheme (C-NKS) is proposed for a

${ }^{1}$ In the simulation section, we used $D_{2}=\mathbf{1}$, where $\mathbf{1}$ is a column vector in which each element is equal to 1 . This corresponds to the case, without loss of generality, where all constraints in Eq. (4) bear the same weight.

${ }^{2}$ Nonnegative residual dispersion is assumed for each link.
PCE-based scenario, and it exploits the NK framework (see Subsection III.B).

- The lightpath establishment with centralized $\ell_{2}$-min framework scheme (C-LMS) is proposed for a PCEbased scenario, and it exploits the $\ell_{2}$-min framework (see Subsection III.B).

In order to guarantee QoT, it is assumed that each node is capable of monitoring $R, P, D$, and $\Phi[15,23,25]$. The following subsections detail the proposed schemes for both distributed and PCE-based path computation scenarios.

\section{A. Distributed Path Computation}

In distributed path computation each ingress node is responsible for the path computation. Each node stores the GMPLS traffic engineering database (TED) with topology and bandwidth information and a local measurement database $(M D B)$ containing the performed end-to-end measurements $\mathbf{R}_{m}, \mathbf{P}_{m}, \mathbf{D}_{m}$, and $\Phi_{m}$. The MDB is filled through signaling protocol extensions [13]. Hence, each node has its own view of the network's physical layer parameters: two different nodes contain information about different sets of lightpaths (i.e., the ones that cross it).

Figure 2 summarizes the D-NKS and D-LMS operations. D-NKS and D-LMS only differ in the exploited QoT estimation framework, which are $\mathrm{NK}$ and $\ell_{2}$-min, respectively. Upon a lightpath request from source $s$ to destination $d\left(L P_{4}\right.$ in Fig. 2), the ingress node $s$ computes a path $q$ to $d$. Then, $s$ exploits its local MDB to estimate the QoT of the lightpath if routed along $q$ ( $1^{\text {st }}$ step). If the QoT parameters of the lightpath, routed along $q$, are already present in the MDB because of previous monitoring, the QoT is computed from data available in the MDB. Otherwise, by applying NK (i.e., D-NKS) or $\ell_{2}$-min (i.e., D-LMS) to the parameters contained in the MDB $\left(\mathbf{R}_{m}, \mathbf{P}_{m}, \mathbf{D}_{m}\right.$, and $\left.\Phi_{m}\right)$, the parameters related to the unobserved lightpath $q$ are estimated and the QoT is derived. If the estimated QoT is unacceptable, another path is computed and the QoT estimation step starts again for the new path. Otherwise ( $2^{\text {nd }}$ step), $s$ starts signaling along $q$ to set up the lightpath ( $3^{\text {rd }}$ step). In the case that the estimated QoT is unacceptable, up to $K$ paths are considered. If each computed path has an estimated unacceptable QoT, the path with the highest QoT is selected. During the signaling session, link resources (i.e., a common wavelength along all links of $q$ ) are reserved and optical cross-connects configured. To verify that the lightpath QoT is acceptable, probing is performed and QoT measurements (all four parameters $R, D, P$, and $\Phi)$ are gathered at $d\left(4^{\text {th }}\right.$ step), which sends the measured values back to $s$ ( $5^{\text {th }}$ step). Each node 
along $q$ (e.g., $s$ in $6^{\text {th }}$ step) fills the MDB entry related to $q$ with the updated end-to-end measurements. If the measured parameters indicate an unacceptable QoT, $s$ releases resources along $q$ and performs another setup attempt. Otherwise, the lightpath is activated and data transmission begins.

\section{B. PCE-Based Path Computation}

In PCE-based computation the PCE stores a database with topology and bandwidth information and a centralized measurement database $(C-M D B)$ containing the end-to-end measurements $\mathbf{R}_{m}, \mathbf{P}_{m}, \mathbf{D}_{m}$, and $\Phi_{m}$ from all the previously attempted lightpath setups. Thus, the PCE has a global view of the past probing measurements. Currently, the IETF is discussing how to maintain information (as updating a database containing QoT information) in databases exploited by the PCE [11]; however, this activity is outside the scope of the paper.

Figure 3 summarizes the C-NKS and C-LMS operations. C-NKS and C-LMS only differ from the exploited QoT estimation framework, which is $\mathrm{NK}$ and $\ell_{2}$-min, respectively. Upon a lightpath request from source $s$ to destination $d\left(L P_{4}\right.$ in Fig. 3), path computation is requested to the PCE $\left(1^{\text {st }}\right.$ step), which computes the path $q$. Then, the C-MDB is exploited to estimate lightpath QoT considering $q$ ( $2^{\text {nd }}$ step). If the C-MDB holds the QoT parameters for the computed path $q$ from previous monitoring, then the QoT is derived from those parameters. Otherwise, by applying NK (i.e., C-NKS) or $\ell_{2}$-min (i.e., C-LMS) to the parameters $\left(\mathbf{R}_{m}, \mathbf{P}_{m}\right.$, $\mathbf{D}_{m}$, and $\Phi_{m}$ ) contained in the C-MDB, the parameters related to $q$ are estimated and the QoT is derived. If the estimated QoT is acceptable ( $3^{\text {rd }}$ step), the PCE sends the computed path information (e.g., list of nodes) to $s$ ( $4^{\text {th }}$ step); otherwise another path is computed. Up to $K$ paths can be considered. In the case that the estimated QoT is unacceptable for each computed path, the path with the highest QoT is selected. Then, $s$ starts signaling along $q$ ( $5^{\text {th }}$ step), and link resources (i.e., a wavelength along $q$ ) are reserved and optical cross-connects configured. To verify that the lightpath QoT is acceptable, probing is performed and QoT measurements are gathered at $d$ ( $6^{\text {th }}$ step). Then, $d$ sends the measured parameters back to $s$ ( $7^{\text {th }}$ step) and updates the measured values in C-MDB ( $8^{\text {th }}$ step). If the measured parameters indicate an unacceptable QoT for $q$, resources are released along $q$ and another setup attempt is performed. Otherwise, the lightpath is activated and data transmission begins.

\section{CASE STUdy}

The proposed lightpath establishment techniques are evaluated by means of a custom event-driven simulator. We consider a Pan-European network (Fig. 4) with 17 nodes and 33 bidirectional links with 40 wavelengths in each direction. $10 \mathrm{Gbit} / \mathrm{s}$ on-off keying (OOK) transmission and direct detection are considered. Fixed worst-case margins accounting for nonmodeled effects are assumed to be $6 \mathrm{~dB}$ as in [13]. Lightpath demands arrive following a Poisson process (average interarrival time: $1 / \lambda$ ) and call durations are exponentially distributed (with mean $1 / \mu=2500 \mathrm{~s}$ ) such that the total offered load in the network, in Erlangs, is $\lambda / \mu$. Upon a lightpath request from source $s$ to destination $d, s$ (if the distributed path computation is utilized) or the PCE (if centralized path computation is utilized) randomly selects a path $q$ within a set $\mathcal{P}(s, d)$ of precomputed paths. $\mathcal{P}(s, d)$ is the set of all paths connecting $s$ and $d$ that are within one hop from the shortest path. Wavelength assignment is first fit.

To evaluate D-NKS, D-LMS and C-NKS, C-LMS, we also disable the estimation frameworks (i.e., NK and $\ell_{2}$-min) and we call the resulting establishment techniques the distributed measurement database-based scheme (D-MDS) and centralized measurement database-based scheme (C-MDS), respectively, in the distributed and in the PCE-based scenarios. With D-MDS and C-MDS, only measurements respectively from the MDB and the C-MDB are used to determine lightpath QoT. Therefore, NK and $\ell_{2}$-min are not exploited with C-MDS and D-MDS to estimate QoT parameters related to nonobserved lightpaths, and we use D-MDS and C-MDS as benchmarks. The actual optical layer performance is emulated considering the model in [13]. The proposed lightpath establishment techniques are compared in terms of the blocking rate after a variable number of setup attempts $n$ : blocking occurs if no wavelength can be found on any path in the set $\mathcal{P}(s, d)$ (i.e., wavelength blocking) or if the monitored QoT parameters (using probing, after establishment) indicate unacceptable lightpath QoT (i.e., QoT blocking). In case of blocking, the successive setup at-

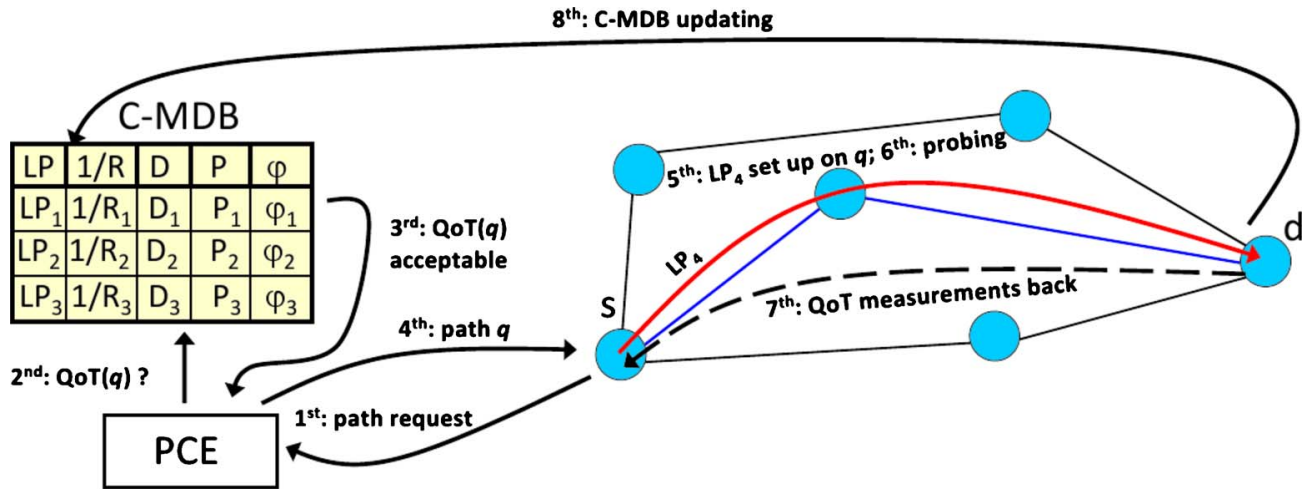

Fig. 3. (Color online) Lightpath establishment operations in a PCE-based scenario with C-NKS and C-LMS. 


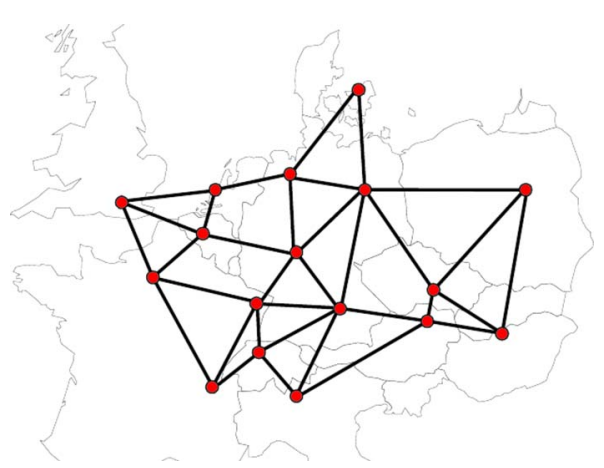

Fig. 4. (Color online) Network topology.

tempt is performed along the maximally link-disjoint path within $\mathcal{P}(s, d)$.

For each scenario (centralized and distributed), we investigate the blocking of the lightpath establishment techniques as a function of time (expressed as the number of lightpath requests), the overall blocking rate versus traffic load, and the path computation time. The blocking behavior as a function of the number of lightpath requests is evaluated for a given load, by establishing a number of lightpaths, and assessing the "instantaneous blocking rate," that is, the blocking rate computed over a small number of lightpath requests using a sliding window average. When the network starts, databases are empty and estimation techniques fail, resulting in a high instantaneous blocking rate. However, as more lightpaths are established, the databases fill in and the estimation frameworks are able to decrease the instantaneous blocking rate compared with cases where no estimation framework is leveraged. The instantaneous blocking rate decreases until converging to a floor, in the steady state.

\section{A. Distributed Path Computation}

In this subsection, D-NKS, D-LMS, and D-MDS are compared. We study the behavior of the blocking rate for each of D-NKS, D-LMS, and D-MDS as new lightpath requests arrive in the network. This is illustrated in Fig. 5. The figure

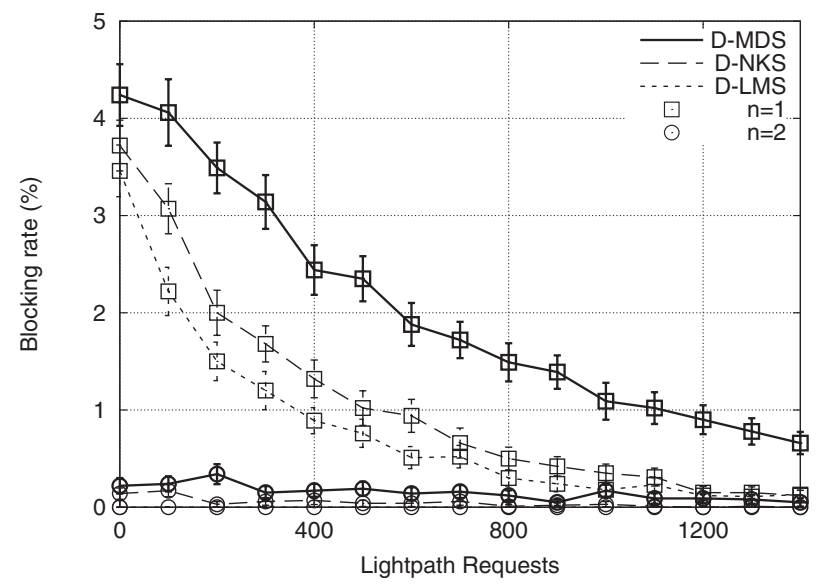

Fig. 5. Temporal evolution of the instantaneous blocking rate after $n \in\{1,2,3\}$ setup attempts in the distributed path computation scenario. shows the "instantaneous blocking rate" obtained for a fixed load (200 Erlangs, low enough that blocking is due to QoT only) after $n \in\{1,2\}$ setup attempts along alternate routes as a function of time, that is, the blocking rate is measured after a varying number of lightpath requests. The plotted results are obtained by averaging 100 randomly generated sequences of lightpath requests and the blocking rate is computed for a sliding window containing the last 100 requests. Up to three setup attempts are admitted for each lightpath request. However, the blocking rate for $n=3$ is not reported since, at this load, the blocking rate is always null. As expected, the instantaneous blocking rate decreases while $n$ increases since further setup attempts along alternative discovery paths are characterized by an acceptable QoT. With each technique, as the MDB is populated, more information is gathered and the instantaneous blocking rate decreases with lightpath demands. Convergence is faster for D-NKS and D-LMS, because they are able to better exploit the information contained in the MDB than D-MDS thanks to the estimation of the end-to-end parameters. D-LMS obtains better performance than D-NKS. For instance, if a single setup attempt is considered, D-MDS achieves a $1 \%$ instantaneous blocking rate after the arrival of 1200 lightpaths, as opposed to only 600 arrivals for D-NKS and 400 for D-LMS.

Figure 6 shows the blocking rate after $n \in\{1,2,3\}$ attempts as a function of the traffic load when 1500 lightpath demands are generated. Each point is obtained by averaging 100 independent trials of 1500 lightpath requests each. In the range [200, 300] Erlangs, only QoT blocking is experienced. In this range, D-MDS and D-NKS experience a constant blocking probability for $n \in\{1,2\}$. Indeed, within 1500 requests, the MDB is not completely filled in the case of $\mathrm{D}-\mathrm{MDS}$, or, in the case of D-NKS, NK does not have enough information to provide confident estimations for every $(s, d)$ pair. D-NKS obtains better performance than D-MDS for $n$ $=1$ and $n=2$. If $n=2$ D-LMS obtains a null blocking within the range of $[200,300]$ Erlangs since $\ell_{2}$-min better exploits information in MDB than NK. For all the techniques and for loads higher than 350 Erlangs, wavelength blocking becomes significant and blocking increases with load.

To clarify the performance of D-MDS, D-NKS, and D-LMS, Table I shows the data related to a single simula-

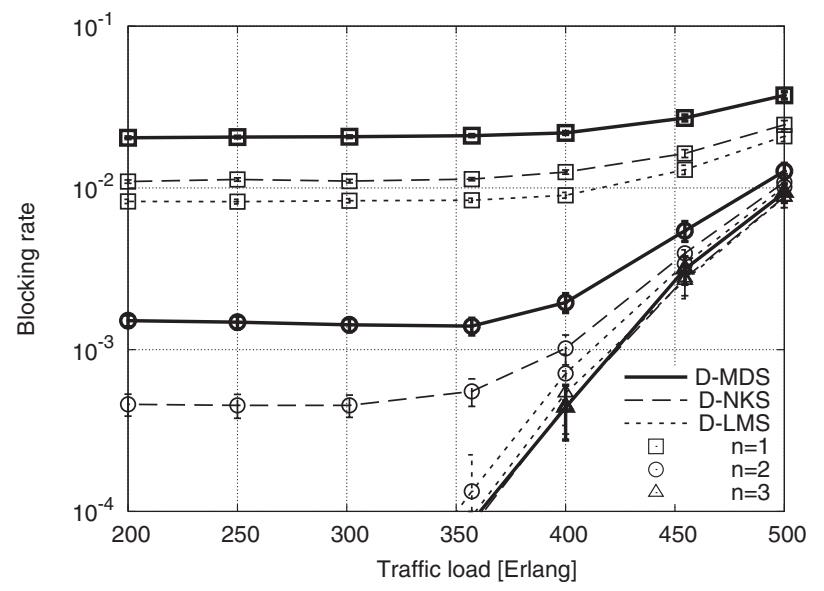

Fig. 6. Blocking rate after $n \in\{1,2,3\}$ setup attempts for a varying load in the distributed path computation scenario. 
TABLE I

Real and Estimated QoT Parameters and Real and Estimated Lightpath QoT for Two Sample Paths When $m_{s}$ Observations Are Available at $s$

\begin{tabular}{|c|c|c|c|c|c|c|c|c|c|c|c|c|c|c|c|c|}
\hline \multirow[b]{2}{*}{$q$} & \multicolumn{3}{|c|}{$1 / R$} & \multicolumn{3}{|c|}{$D[\mathrm{ps} / \mathrm{nm}]$} & \multicolumn{3}{|c|}{$P^{2}\left[\mathrm{ps}^{2}\right]$} & \multicolumn{3}{|c|}{$\Phi$} & \multirow[b]{2}{*}{$\begin{array}{l}\text { real } \\
\text { QoT }\end{array}$} & \multicolumn{3}{|c|}{$\begin{array}{c}\text { Estimated } \\
\text { QoT }\end{array}$} \\
\hline & real & NK & $\begin{array}{l}\ell_{2^{-}} \\
\min \end{array}$ & real & NK & $\begin{array}{l}\ell_{2^{-}} \\
\min \end{array}$ & real & NK & $\begin{array}{c}\ell_{2^{-}} \\
\min \end{array}$ & real & NK & $\begin{array}{c}\ell_{2^{-}} \\
\min \end{array}$ & & $\begin{array}{c}\text { D- } \\
\text { MDS }\end{array}$ & $\begin{array}{c}\text { D- } \\
\text { NKS }\end{array}$ & $\begin{array}{l}\text { D- } \\
\text { LMS }\end{array}$ \\
\hline $\begin{array}{c}q_{1} \\
m_{s}=6\end{array}$ & 0.034 & 0.007 & 0.053 & 41 & 10.25 & 26.98 & 92.99 & 21.28 & 49.25 & 0.044 & 0.009 & 0.064 & acc. & none & acc. & un. \\
\hline $\begin{array}{c}q_{2} \\
m_{s}=6\end{array}$ & 0.040 & 0.007 & 0.06 & 51.25 & 10.25 & 32.55 & 99.7 & 21.28 & 58.57 & 0.04 & 0.009 & 0.08 & un. & none & acc. & un. \\
\hline $\begin{array}{c}q_{1} \\
m_{s}=34\end{array}$ & 0.034 & 0.026 & 0.046 & 41 & 31.12 & 34.23 & 92.99 & 71.18 & 74.7 & 0.044 & 0.034 & 0.050 & acc. & none & acc. & acc. \\
\hline $\begin{array}{c}q_{2} \\
m_{s}=40\end{array}$ & 0.040 & 0.040 & 0.040 & 51.25 & 51.25 & 51.25 & 99.78 & 99.78 & 99.78 & 0.047 & 0.047 & 0.047 & un. & none & un. & un. \\
\hline
\end{tabular}

tion experiment. Table I contains the real and the estimated values of each QoT parameter and the real and the estimated lightpath QoT (i.e., acceptable or unacceptable) for two sample paths. In particular, Table I aims to i) show when a setup attempt is performed along paths with a real unacceptable QoT and thus when a further setup attempt is required and ii) provide a qualitative understanding of the better performance achieved by D-LMS with respect to D-NKS in terms of blocking probability. The two sample paths $q_{1}$ and $q_{2}$, which have not been monitored, connecting the same pair $(s, d)$ are considered. $m_{s}$ lightpaths traversing $s$ have been observed and related parameters are stored in the MDB at node $s$. The real QoT of $q_{1}$ and $q_{2}$ are acceptable and unacceptable, respectively. With D-MDS no estimation is performed and probing is always performed along the computed path. In this case a further setup attempt is required if the attempted path is $q_{2}$ with D-MDS. On the contrary, the lightpath is established if the chosen path is $q_{1}$. When few entries are available in the local MDB (e.g., when $m_{s}=6$ ), the QoT is estimated to be acceptable with NK (i.e., probing is performed with D-NKS) for both $q_{1}$ and $q_{2}$, and thus a further setup attempt is required if $q_{2}$ is first selected. In the same scenario (i.e., when $m_{s}=6$ ), $\ell_{2}$-min tends to be more pessimistic and QoT is estimated to be unacceptable for both $q_{1}$ and $q_{2}$. In this case, the path with the higher QoT ( $q_{1}$ in this case) is probed, and no further setup attempt is required with D-LMS. Estimation accuracy increases with the number of observations (e.g., with $\mathrm{NK} 1 / R$ is estimated to be 0.007 and 0.026 , when $m=6$ and $m_{s}=34$, respectively, while the actual $1 / R$ is 0.034$)$. For instance, when the local MDB includes numerous entries (e.g., $m_{s}=40$ ) also with D-NKS, probing is avoided along $q_{2}$, which presents actual unacceptable QoT.

The path computation time required by each scheme has been evaluated by considering the actual path computation time experienced during the simulations presented in Fig. 5. The simulations have been run on a Pentium Intel Q8200 at $2.33 \mathrm{GHz}$. With D-MDS, the path computation time can be considered negligible (less than $10^{-5} \mathrm{~s}$ ). On average, in the case of D-NKS and D-LMS, a lightpath path computation requires 0.4 and $107 \mathrm{~ms}$, respectively. It is expected that the path computation time in a real transparent network does not increase since all-optical networks with a significantly higher number of nodes and links are unlikely to be set up because of impairment accumulation along lightpaths.

\section{B. Centralized PCE-Based Path Computation}

In this subsection, C-NKS, C-LMS, and C-MDS are compared. As before, we first study the behavior of the instantaneous blocking rate for each of C-NKS, C-LMS, and C-MDS. Figure 7 shows the behavior of the instantaneous blocking rate for C-NKS, C-LMS, and C-MDS as new lightpath requests arrive in the network. Up to $n=3$ lightpath establishment attempts are allowed. The total offered load is set to a low value, 200 Erlangs, such that wavelength blocking is negligible compared with QoT blocking. As new demands arrive, the C-MDB is populated, allowing C-MDS to know the QoT for an increasing number of lightpaths and allowing C-NKS and C-LMS to estimate QoT more accurately, resulting in a decrease of the instantaneous blocking rate even for $n=1$. For instance, C-NKS and C-LMS obtain a null blocking with $n=1$ after 300 lightpath requests, while C-MDS takes 1400 lightpath requests and a second attempt $(n=2)$ per lightpath demand to achieve a null blocking.

Figure 8 shows the establishment of 1500 lightpaths for a varying offered load in the network after $n \in\{1,2,3\}$ setup

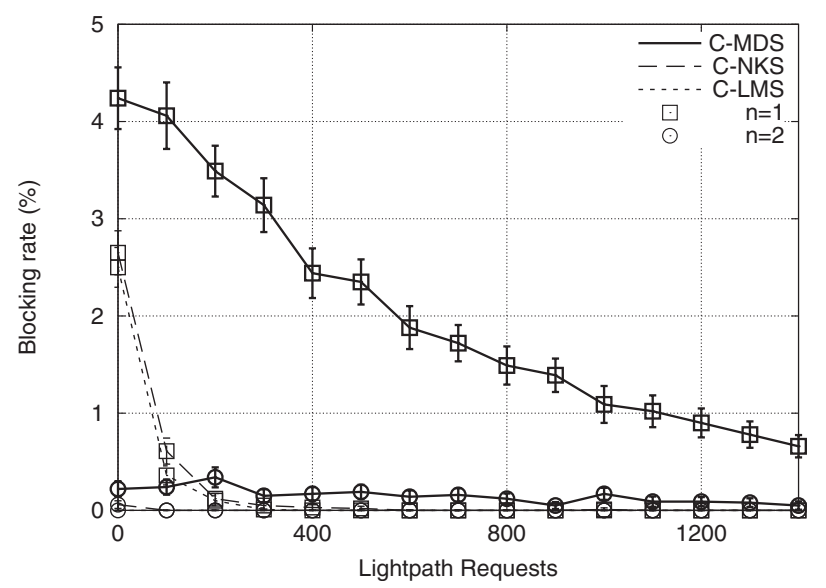

Fig. 7. Temporal evolution of the instantaneous blocking rate after $n \in\{1,2,3\}$ setup attempts in the PCE-based scenario. 


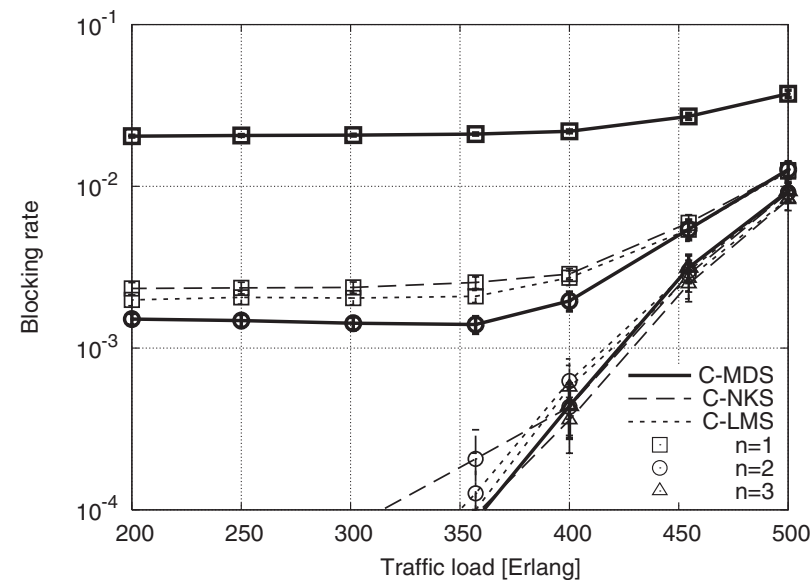

Fig. 8. Blocking rate after $n \in\{1,2,3\}$ setup attempts for a varying load in the PCE-based scenario.

attempts. The blocking rate decreases when the allowed number of setup attempts increases. Blocking increases with traffic load since wavelength blocking contributes at high loads. Contrary to C-MDS, C-NKS and C-LMS leverage the estimation frameworks and are seen to strongly decrease the number of required setup attempts to successfully establish lightpaths, compared with C-MDS. In particular, the blocking probability is much lower with C-NKS and C-LMS than C-MDS for $n=1$. C-MDS obtains with $n$ $=3$ the same blocking probability that C-NKS and C-LMS obtain with $n=2$. For instance, for a load of 400 Erlangs, C-MDS requires three lightpath setup attempts to achieve a blocking rate lower than $10^{-3}$, while C-NKS and C-LMS require only two attempts.

To assess the path computation time, we implemented the simulated PCE on the same PC of the previous section. With C-MDS, path computation time can be considered negligible as in the distributed scenario. On average, in the case of C-NKS and C-LMS, a lightpath path computation requires 103 and $137 \mathrm{~ms}$, respectively. In terms of absolute values, a centralized PCE is expected to operate on powerful dedicated CPUs, thus potentially increasing the computational speed with respect to the distributed case.

\section{Distributed Versus Centralized PCE-Based Scenarios}

Table II reports an extract of Figs. 5 and 7. The comparison between the distributed and the PCE-based centralized schemes highlights that without QoT parameter estimation

TABLE II

Blocking Rate (\%) After $n$ Setup Attempts AND $k$ LightPATH REQUESTS

\begin{tabular}{cccccc}
\hline$n$ & $k$ LP requests & C-MDS & D-MDS & C-LMS & D-LMS \\
\hline 1 & 100 & 4.06 & 4.06 & 0.36 & 2.22 \\
1 & 400 & 2.42 & 2.44 & 0 & 0.89 \\
2 & 100 & 0.23 & 0.24 & 0 & 0 \\
2 & 400 & 0.17 & 0.17 & 0 & 0 \\
\hline
\end{tabular}

(i.e., D-MDS and C-MDS) no advantage is provided in terms of blocking probability by the centralized knowledge of QoT information. Indeed, with D-MDS and C-MDS, just the QoT parameters (if available) of the selected lightpath (by the ingress or by the PCE, respectively) are exploited. Only if the lightpath has been already monitored, such information is available with both schemes. Blocking probability results confirm that, at different setup attempts or upon different amount of offered lightpath requests, D-MDS and C-MDS provide the same results. On the contrary, if QoT parameter estimation is introduced, e.g., when $\ell_{2}$-min framework is applied, results show that at the first setup attempt (i.e., $n$ $=1$ ), the C-LMS scheme provides significant advantage with respect to D-LMS by exploiting a larger amount of QoT parameter information. For example, as shown in Table II, upon 100 requests D-LMS and C-LMS provide a blocking rate of $2.22 \%$ and $0.36 \%$, respectively. Upon 400 requests, the two schemes still perform differently, achieving a null blocking rate and a blocking less than $1 \%$ with C-LMS and D-LMS, respectively. In addition, as early as at the second setup attempt (i.e., $n=2$ ), both C-LMS and D-LMS achieve optimal results, i.e., null blocking rate.

\section{CONCLUSIONS}

By combining monitoring hardware and end-to-end estimation frameworks, we showed that it is possible to estimate the QoT for a lightpath before it is established, thereby avoiding setup attempts for lightpaths with (estimated) low QoT. We investigated the utilization of two end-to-end estimation frameworks: network kriging and norm $\ell_{2}$ minimization. The exploitation of these estimation frameworks during lightpath establishment may be applied for both distributed path computation and centralized PCE-based network scenarios. A practical consequence of the proposed techniques is that, for a given target blocking rate, fewer establishment attempts are required thanks to the estimation step. For instance, in a distributed network scenario, the utilization of end-to-end estimation decreases the number of required establishment attempts from three to two to achieve a given target blocking rate of $10^{-3}$. We also showed that the lightpath establishment schemes assisted with endto-end estimation converge faster in terms of blocking rate than the schemes where no end-to-end estimation is performed. This has an important practical implication: estimation-based schemes are more reactive to assist dynamic transparent networks of the future. We observed that the exploitation of the $\ell_{2}$-min yields better performance than network kriging in terms of blocking probability, particularly in the case of the distributed scenario. In a PCEbased scenario, the exploitation of network kriging or norm $\ell_{2}$ minimization induces more benefits in terms of blocking probability than with the distributed scenario when limited QoT information is available. Indeed, a centralized database holds significantly more information that can be better exploited by the estimation frameworks than in the distributed scenario, thus reducing the average amount of required lightpath setup attempts. However, particularly in the case of network kriging, better performance in terms of required path computation time is achieved in the distributed with 
respect to the centralized scenario. In the centralized PCEbased scenario the average performance in terms of required path computation time is comparable between network kriging and $\ell_{2}$-min, while in the distributed scenario network kriging outperforms the other one.

\section{ACKNOWLEDGMENT}

The work described in this paper was carried out with the support of the BONE project ("Building the Future Optical Network in Europe"), a Network of Excellence funded by the European Commission through the 7th ICT-Framework Programme and DICONET projects. This paper is an extended version of the work presented in $[35,36]$.

\section{REFERENCES}

[1] I. Tomkos, S. Azodolmolky, M. Angelou, D. Klonidis, Y. Ye, C. V. Saradhi, E. Salvadori, A. Zanardi, and R. Piesiewicz, "Impairment aware networking and relevant resiliency issues in alloptical networks," in 34th European Conf. on Optical Communication (ECOC), 2008.

[2] O. Tamm, "Scaling networks considering power trends," in 35th European Conf. on Optical Communication (ECOC), Workshop WS4, Vienna, Austria, 2009.

[3] B. Ramamurthy, D. Datta, H. Feng, J. Heritage, and B. Mukherjee, "Impact of transmission impairments on the teletraffic performance of wavelength-routed optical networks," $J$. Lightwave Technol., vol. 17, no. 10, pp. 1713-1723, Oct. 1999.

[4] A. Farrel, J.-P. Vasseur, and J. Ash, "A path computation element (PCE)-based architecture," IETF RFC 4655, Aug. 2006.

[5] S. Azodolmolky, M. Klinkowski, E. Marin, D. Careglio, J. SolePareta, and I. Tomkos, "A survey on physical layer impairments aware routing and wavelength assignment algorithms in optical networks," Comput. Netw., vol. 53, no. 7, pp. 926944, May 2009.

[6] S. Pachnicke, J. Reichert, S. Spalter, and E. Voges, "Fast analytical assessment of the signal quality in transparent optical networks," J. Lightwave Technol., vol. 24, no. 2, pp. 815-824, Feb. 2006.

[7] A. Morea, N. Brogard, F. Leplingard, J.-C. Antona, T. Zami, B. Lavigne, and D. Bayart, "QoT function and A* routing: an optimized combination for connection search in translucent networks," J. Opt. Netw., vol. 7, no. 1, pp. 42-61, 2008.

[8] T. Deng, S. Subramaniam, and J. Xu, "Crosstalk-aware wavelength assignment in dynamic wavelength-routed optical networks," in 1st Int. Conf. on Broadband Networks (BROADNETS), 2004.

[9] P. Kulkarni, A. Tzanakaki, C. Mas Machuka, and I. Tomkos, "Benefits of Q-factor based routing in WDM metro networks," in 31st European Conf. on Optical Communication (ECOC), 2005, pp. 981-982.

[10] P. Pavon-Mariño, S. Azodolmolky, R. Aparicio-Pardo, B. Garcia-Manrubia, Y. Pointurier, M. Angelou, J. Sole-Pareta, J. Garcia-Haro, and I. Tomkos, "Offline impairment aware RWA algorithms for cross-layer planning of optical networks," $J$. Lightwave Technol., vol. 27, no. 12, June 2009.

[11] Y. Lee and G. Bernstein, "Alternative approaches to traffic engineering database creation and maintenance for path computation elements," IETF draft, May 2009.

[12] J. Strand, A. Chiu, and R. Tkach, "Issues for routing in the optical layer," IEEE Commun. Mag., vol. 39, no. 2, pp. 81-87, Feb. 2001.

[13] F. Cugini, N. Sambo, N. Andriolli, A. Giorgetti, L. Valcarenghi,
P. Castoldi, E. Le Rouzic, and J. Poirrier, "Enhancing GMPLS signaling protocol for encompassing quality of transmission (QoT) in all-optical networks," J. Lightwave Technol., vol. 26, no. 19, pp. 3318-3328, Oct. 2008.

[14] G. Martinelli and A. Zanardi, "GMPLS signaling extensions for optical impairment aware lightpath setup," IETF draft, July 2009.

[15] D. C. Kilper, R. Bach, D. J. Blumenthal, D. Einstein, T. Landolsi, L. Ostar, M. Preiss, and A. E. Willner, "Optical performance monitoring," J. Lightwave Technol., vol. 22, no. 1, Jan. 2004.

[16] N. Sambo, F. Cugini, I. Cerutti, L. Valcarenghi, P. Castoldi, J. Poirrier, E. Le Rouzic, and C. Pinart, "Probe-based schemes to guarantee lightpath quality of transmission QoT in transparent optical networks," in 34th European Conf. on Optical Communication (ECOC), 2008.

[17] Y. Pointurier, M. Coates, and M. Rabbat, "Active monitoring of all-optical networks," in 10th Int. Conf. on Transparent Optical Networks (ICTON), 2008.

[18] D. B. Chua, E. D. Kolaczyk, and M. Crovella, "Network kriging," IEEE J. Sel. Areas Commun., vol. 24, no. 12, pp. 22632272, Dec. 2006.

[19] H. Song, L. Qiu, and Y. Zhang, "NetQuest: a flexible framework for large-scale network measurement," IEEE/ACM Trans. Netw., vol. 17, pp. 106-119, Feb. 2009.

[20] M. J. Coates, A. O. Hero III, R. Nowak, and B. Yu, "Internet tomography," IEEE Signal Process. Mag., pp. 47-65, May 2002.

[21] N. Sambo, F. Cugini, M. Secondini, G. Bottari, F. Cavaliere, P. Iovanna, and P. Castoldi, " $10-40 \mathrm{Gbit} / \mathrm{s}$ multi bit-rate GMPLS-controlled transparent optical networks in presence of cross-phase modulation," in 36th European Conf. on Optical Communication (ECOC), 2010.

[22] J.-C. Antona, S. Bigo, and J.-P. Faure, "Nonlinear cumulated phase as a criterion to assess performance of terrestrial WDM systems," in Optical Fiber Communication Conf. (OFC), 2002, paper WX5.

[23] Agiltron, "In-band impairment detector" [Online]. Available: http://www.agiltron.com/pdfs/impairment\%20detector.pdf.

[24] "Diconet deliverable 3.1" [Online]. Available: http:// www.diconet.eu/.

[25] M. Petersen, N. Sambo, N. Andriolli, and M. Scaffardi, "PMD monitoring using optical sideband filtering," in 17th Annu. Meeting of the IEEE Lasers and Electro-Optics Society (LEOS), 2009, pp. 129-130.

[26] B.-W. Hakki, "Polarization mode dispersion compensation by phase diversity detection," IEEE Photon. Technol. Lett., vol. 9, no. 1, pp. 121-123, June 1997.

[27] Y. K. Lize, L. Christen, J.-Y. Yang, P. Saghari, S. Nuccio, A. E. Willner, and R. Kashyap, "Independent and simultaneous monitoring of chromatic and polarization-mode dispersion in OOK and DPSK transmission," IEEE Photon. Technol. Lett., vol. 19, no. 1, pp. 3-5, Jan. 2007.

[28] S. Wielandy, M. Fishteyn, T. Her, D. Kudelko, and C. Zhang, "Dispersion monitoring and automatic compensation based on differential nonlinear detection scheme," in Optical Fiber Communication Conf. (OFC), 2003, paper TuD5.

[29] Y. Takushima, H. Yoshimi, Y. Ozeki, K. Kikuchi, H. Yamauchi, and H. Taga, "In-service dispersion monitoring in 32 $\times 10.7$ Gbps WDM transmission system over transatlantic distance using optical frequency-modulation method," J. Lightwave Technol., vol. 22, no. 1, pp. 257-265, 2004.

[30] Q. Yu, Z. Pan, L. Yan, and A. E. Willner, "Chromatic dispersion monitoring technique using sideband optical filtering and clock phase-shift detection," J. Lightwave Technol., vol. 20, no. 12, pp. 2267-2271, 2002.

[31] J.-C. Antona, S. Bigo, and S. Kosmalski, "Nonlinear index measurements of various fibre types over $\mathrm{C}+\mathrm{L}$ bands using four- 
wave mixing," in 27th European Conf. on Optical Communication (ECOC), 2001, pp. 270-271.

[32] Y. Namihira, A. Miyata, and N. Tanahashi, "Nonlinear coefficient measurements for dispersion shifted fibres using selfphase modulation method at 1.55pm," Electron. Lett., vol. 30, no. 14, pp. 1171-1172, 1994.

[33] M. Saunders, "Primal-dual interior method for convex objectives" [Online]. Available: http://www.stanford.edu/group/SOL/ software/pdco.html.

[34] S. Canu and C. Ong, "Learning and regularization from interpolation to approximation" [Online]. Available: http://asi.insarouen.fr/enseignants/scanu/Cours2.ps.

[35] N. Sambo, Y. Pointurier, F. Cugini, P. Castoldi, and I. Tomkos, "Lightpath establishment in PCE-based dynamic transparent optical networks assisted by end-to-end quality of transmission estimation," in 11th Int. Conf. on Transparent Optical Networks (ICTON), Island of São Miguel, Azores, Portugal, 2009.

[36] N. Sambo, Y. Pointurier, F. Cugini, L. Valcarenghi, P. Castoldi, and I. Tomkos, "Lightpath establishment in distributed transparent dynamic optical networks using network kriging," in 35th European Conf. on Optical Communication (ECOC), Vienna, Austria, 2009.

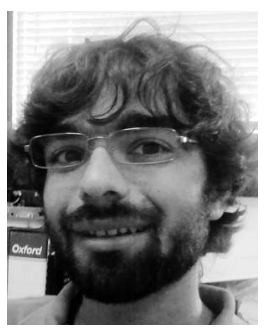

Nicola Sambo received the Laurea degree cum laude in telecommunication engineering from the University of Pisa, Italy, in 2004 and the Ph.D. degree from Scuola Superiore Sant'Anna, Pisa, Italy in the "Center of Excellence for Information, Communication and Perception Engineering (CEIICP)" in 2009. He was a visiting student at France Télécom, Lannion, France, and at Athens Information Technology, Greece. He is also collaborating with the National Photonic Networks Laboratory of the CNIT in Pisa and Ericsson, Pisa, Italy. His research interests include WDM network planning and modeling, quality of transmission, QoS and fault tolerance in WDM optical networks, GMPLS protocol suite for optical networks, routing, and setup.

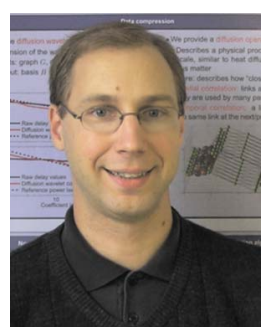

Yvan Pointurier (S'02-M'06) received a Diplôme d'Ingénieur from Ecole Centrale de Lille, France, in 2002, a M.S. in computer science in 2002, and a Ph.D. in electrical engineering in 2006, both from the University of Virginia, USA. He spent two years at McGill University in Montreal, Canada, as a Postdoctoral Fellow and then one year at Athens Information Technology, Greece, as a Technical Leader for a European Project. In 2009 he joined Alcatel-Lucent Bell Labs, France, as a Research Engineer. His research interests span design, optimization, and monitoring of networks in general and optical networks in particular. Dr. Pointurier is a co-recipient of the Best Paper Award at the IEEE ICC 2006 Symposium on Optical Systems and Networks.



Filippo Cugini received the M.S. degree in telecommunication engineering from the University of Parma, Italy. Since 2001, he has been with the National Laboratory of Photonic Networks, Consorzio Nazionale Interuniversitario per le Telecomunicazioni (CNIT), Pisa, Italy. In 2010, he co-chaired the Optical Network and System (ONS) symposia at the IEEE Globecom Conference. His main research interests include MPLS, GMPLS, and PCE protocols and architectures; survivability in IP over WDM networks; and traffic engineering in grid networking. He is co-author of 6 international patents and more than 60 IEEE publications.

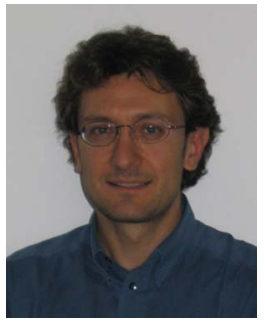

Luca Valcarenghi holds a Laurea degree in electronics engineering (1997) from the Politecnico di Torino, Italy, an M.S. in electrical engineering (1999), and a Ph.D. in electrical engineering-telecommunications (2001), both from the University of Texas at Dallas (UTD). Between January 2002 and August 2002 he was a Research Associate of the Optical Networking Advanced Research (OpNeAR) Lab of the University of Texas at Dallas Erik Jonsson School of EE/CS. Since September 2002 he has been an Assistant Professor at the Scuola Superiore Sant'Anna of University Studies and Doctoral Research of Pisa, Italy. Dr. Valcarenghi has published more than 100 papers in international journals and conference proceedings and actively participated in the TPC of Globecom and the ICC Optical Network and System Symposium and Next-Generation Networking \& Internet Symposium. His main research interests are design, analysis, and optimization of optical networks; artificial intelligence optimization techniques; reliability of communication networks; IP over WDM networking; QoS in network infrastructures for grid computing; fixed and mobile network integration; fixed network backhauling for mobile networks; and energy efficiency in communications networks.

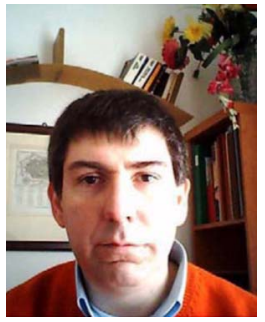

Piero Castoldi (Ph.D. in information technology) has been an Associate Professor at Scuola Superiore Sant'Anna, Pisa, Italy, since 2001. He spent abroad at Princeton University (USA) overall about two years in 1996, 1997, 1999, and 2000, and in 2001 he visited for two months the University of Texas at Dallas, USA. He has also served as a Project Manager of many projects of the Inter-universitary National Consortium for Telecommunications (CNIT), and since January 2005 he has been Director of the CNIT National Laboratory of Photonic Networks. He is also currently Leader of the "Networks and Services" research area at the Center of Excellence for Networks Engineering at Scuola Superiore Sant'Anna. His research interests cover telecommunications networks and systems, both wired and wireless, and more recently reliability, switching paradigms, and control of optical networks, including applicationnetwork cooperation mechanisms, in particular for grids and clouds. He is an IEEE Member and he is author of more than 200 publications in international journals and conference proceedings.

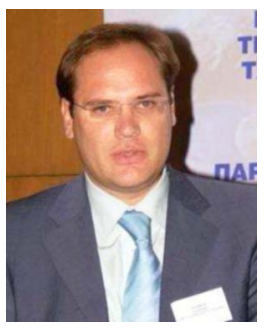

Ioannis Tomkos has received the prestigious title of "Distinguished Lecturer" of the IEEE Communications Society for the topic of transparent optical networking. Together with his colleagues and students he has authored about 320 peer-reviewed archival articles (over 170 IEEE-sponsored), including about 100 journal/magazine/book publications (about 60 IEEE-sponsored). Dr. Tomkos has served as the Chair of the International "Optical Networking" Technical Committee of the IEEE Communications Society and the Chairman of the IFIP working group on "Photonic Networking." He is currently the Chairman of the OSA Technical Group on Optical Communications. He has been General Chair, Technical Program Chair, Subcommittee Chair, Symposium Chair, and/or a member of the steering/organizing committees for the major conferences (e.g., OFC, ECOC, IEEE Globecom, IEEE ICC, ONDM, etc.) in the area of telecommunications/networking (about 100 conferences/ symposia/workshops). In addition he is a member of the Editorial Boards of the IEEE/OSA Journal of Lightwave Technology, the IEEE/OSA Journal of Optical Communications and Networking, the IET Journal on Optoelectronics, and the International Journal on Telecommunications Management. He is a Fellow of the IET. 